Revista Colombiana de Obstetricia y Ginecología Vol. 55 No.4 • $2004 \cdot(279-286)$

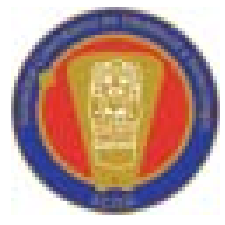

InVESTIGACión Original

\title{
CARACTERIZACIÓN DE LOS TRASTORNOS HIPERTENSIVOS DEL EMBARAZO*
}

\author{
CHARACTERISING HYPERTENSIVE DISORDERS DURING PREGNANCY \\ IN VILLAVICENCIO, COLOMBIA.* \\ Juan Álvaro Salazar, M.D.**, Juan Carlos Triana, M.D.***, \\ Franklyn Edwin Prieto, M.D.****
}

Recibido: mayo 24/2004 - Revisado: julio 19/2004 - Aceptado: noviembre 8/2004

\section{RESUMEN}

Objetivo: describir la incidencia de los trastornos hipertensivos del embarazo en Villavicencio (Colombia) y las características que pueden relacionarse con la hospitalización materna y las complicaciones.

Materiales y métodos: registro prospectivo de embarazadas hipertensas, mediante búsqueda activa. Se registraron las características sociodemográficas, clínicas y de tratamiento; los datos son comparados con información institucional de la población de embarazadas atendidas en el periodo de estudio. Las participantes del grupo de hipertensas fueron clasificadas con base en la ausencia o presencia de complicaciones y hospitalización materna. Se hizo comparación de los grupos mediante análisis univariado y regresión logística múltiple.

Resultados: la incidencia de trastornos hipertensivos del embarazo fue de 5,2 (IC95\%: 4,2 6,3\%). La edad y el número de gestaciones entre las hipertensas y la población total de embarazadas, no mostraron diferencias. Se encontraron mayores porcentajes de parto pretérmino, parto intervenido y peso inferior a $2.500 \mathrm{gr}$ en el grupo de hipertensas. El 53,2\% de los casos se clasificaron

Investigación realizada con el auspicio de la Facultad de Medicina de la Universidad Cooperativa y del Convenio Clínica Martha-Saludcoop. Villavicencio, Colombia

** Ginecólogo Obstetra, Epidemiólogo, Universidad Cooperativa.

Correo electrónico: jasalaza@hotmail.com

*** Epidemiólogo. Saludcoop EPS.

**** MSc SP, Epidemiólogo. Universidad de los Llanos. como complicados, el 15,4\% de ellos no recibió un tratamiento específico. Se encontró asociación entre más de 4 días de hospitalización materna con proteinuria grave y epigastralgia. El parto pretérmino y la proteinuria leve fueron predictores de complicaciones. La epigastralgia fue pronóstico para complicación con una razón de probabilidades positiva de 2,29 y negativa de 0,51, índice de Youden de 0,53.

Conclusiones: la hipertensión en el embarazo posee alto impacto en la salud materna y perinatal. Se sugiere fortalecer las medidas de prevención, diagnóstico y los recursos para tratamiento.

Palabras clave: eclampsia, preeclampsia, proteinuria complicaciones del embarazo.

\section{SUMMARY}

Objective: describing the incidence of hypertensive disorders during pregnancy in Villavicencio (Colombia) and features which may be related to complications or hospitalisation.

Materials and methods: an active search was made of hypertense pregnancies; a prospective record was kept of treatment and socio-demographic and clinical characteristics. This data was compared with institutional information regarding the pregnant population attended during the study. Hypertense group variables were compared with the presence or absence of complications and hospitalisation during this time using a multiple logistic regression model. 
Results: there were 5.2\% (95\% CI: 4.2\%-6.3\%) pregnant hypertensive disorders. Comparing hypertense patients' age and their number of gestations with the total population of pregnant women did not reveal statistically significant differences. Greater percentages of pre-term birth, assisted delivery and infant weight being below 2,500gm were found in the hypertense group. $53.2 \%$ of the cases were classified as being complicated and $15.4 \%$ of them did not receive specific treatment for this condition. There was an association between more than 4 days' hospitalisation with severe proteinuria and epigastralgia. Pre-term pregnancy and mild proteinuria were taken as complication predictors. Epigastralgia was used for predicting complications, having a 2.29 likelihood ratio and 0.51 negative ratio, with 0.53 Youden index.

Conclusions: hypertension during pregnancy has a great impact on maternal and perinatal health. It is suggested that preventative measures should be strengthened, as should early diagnosis and treatment of complications during pregnancy.

Key words: eclampsia, preeclampsia, proteinuria, pregnancy complications.

\section{INTRODUCCIÓN}

Es reconocida la importancia de los desórdenes hipertensivos en la gestación por su incidencia y como causa de complicaciones de la salud maternofetal. La incidencia reportada varía entre 3 y 14\%. ${ }^{1-4}$ En poblaciones con condiciones socioeconómicas y políticas críticas, como las que se presentan en Colombia, es lógico que se generen cambios demográficos y de acceso a los servicios que afectan directamente el comportamiento de los procesos de salud-enfermedad.

La hipertensión en el embarazo se constituye en un grupo complejo de patologías clasificadas en grupos aparentemente bien definidos, con criterios que deben ser evaluados y adaptados al contexto para su aplicación, según la recomendación. ${ }^{5}$

La modificación de los parámetros diagnósticos puede afectar los resultados en estudios de fre- cuencia; más aún, si la presencia de un síntoma varía su clasificación, generando cambios de acuerdo a la percepción de la paciente o su médico, es más difícil establecer el diagnóstico, clasificación y manejo de las pacientes ante la ausencia de proteinuria.

El sistema de vigilancia en salud pública (Sivigila) de Colombia ha incluido la muerte materna y perinatal como eventos sujetos de control, pero sería necesario agregar el registro de eventos estrechamente relacionados como la hipertensión en la embarazada, para intervenirlos oportuna y eficazmente.

Dado que la hipertensión complica frecuentemente la gestación, el objeto del presente estudio fue establecer su magnitud regional de acuerdo a los nuevos parámetros de clasificación, los factores relacionados con su etiología y pronóstico, incluyendo los síntomas y los esquemas de tratamiento empleados.

\section{MATERIALES Y MÉTODOS}

Se presenta un estudio descriptivo longitudinal prospectivo realizado mediante registro institucional de casos de hipertensión arterial en la gestación, en las cinco instituciones de mayor cobertura para atención del parto en Villavicencio (Colombia), durante un periodo de 90 días. Cuatro de ellas atienden todos los partos de las beneficiarias de las empresas a las que prestan servicios; sólo el Hospital Departamental es centro de referencia de primero y segundo nivel.

El registro institucional se efectuó diariamente mediante búsqueda activa de casos entre las gestantes que asistían para la atención del parto; se consideró como hipertensa, la gestante que desde su captación en los servicios de salud o durante su hospitalización presentó una tensión arterial mayor de 140/90 verificable y que finalizó su embarazo sin establecer ningún criterio de exclusión. A las gestantes captadas se le explicó el propósito del estudio y la confidencialidad de la información solicitada. Una vez hecha y entendida la explicación las pacientes decidían su participación. Se adquirió el compromiso de no discriminar la información por entidades participantes.

En los casos detectados se obtuvo información mediante un formato estandarizado a partir de dos 
fuentes: encuesta directa para detectar antecedentes sociodemográficos, médicos y ginecoobstétricos reconocidos como factores de riesgo para hipertensión, ${ }^{1-9}$ y datos de la historia clínica institucional sobre evolución, manejo clínico, resultados de laboratorio, tipo de parto, características del recién nacido y complicaciones de la gestación o el parto.

Las variables de diagnóstico, clasificación y evolución de cada caso se registraron de manera cronológica con base en su realización o detección, y se estratificaron con los rangos aceptados para diagnóstico o definir gravedad; $;^{1-5}$ entre éstos se tomó la deshidrogenasa láctica mayor de $500 \mathrm{mU} / \mathrm{L}$ y la aspartato-amino-transferasa mayor de $70 \mathrm{U} / \mathrm{L}$.

Con los datos que fue posible recolectar de los registros de cada institución, se obtuvo información de las mujeres no hipertensas atendidas por parto durante el periodo del estudio (población no encuestada) así: edad materna en 1.078, edad gestacional en 1.383, número de embarazos en 1.026, número de controles prenatales en 635, vía del parto en 1.695 nacimientos y peso al nacer en 1.202 partos.

Para el análisis, en primera instancia, a partir del número total de partos institucionales se estableció la incidencia de hipertensión y con la información de la encuesta, sus características y evolución. Luego se compararon las características de las gestantes hipertensas con la información recolectada en las instituciones del resto de las gestantes.

En una segunda parte del estudio, las gestantes hipertensas se clasificaron en complicadas y no complicadas, y luego por hospitalización mayor y menor de cuatro días.

Para la clasificación como complicadas se tuvo en cuenta la aparición de los signos paraclínicos que definen la gravedad de la preeclampsia y la presencia de complicaciones clínicas maternas o perinatales, tomadas por los diagnósticos o tratamientos descritos en la historia clínica; se aceptaron los criterios de cada institución o del médico tratante, para eventos como anemia grave, choque, coagulopatía, insuficiencia renal, insuficiencia respiratoria en un caso; o restricción de crecimiento intrauterino, oligoamnios, sufrimiento fetal agudo, dificultad respiratoria del recién nacido, en el otro.

De esta manera se conformaron dos grupos así:

No complicados: los casos de hipertensión gestacional e hipertensión crónica sin complicaciones clínicas ni paraclínicas y los de preeclampsia leve o moderada.

Complicados: los casos de hipertensión gestacional con complicación clínica o paraclínica, los de preeclampsia grave y todos los de hipertensión crónica con preeclampsia o complicación sobreagregadas.

La inclusión en el grupo de complicaciones y los días de hospitalización materna mayores de cuatro días se establecieron como variables dependientes para la regresión logística múltiple. Se utilizó una combinación de métodos de construcción de modelos entre introducir datos (enter), de descarte (backward) y de inclusión (forward). El estadístico de prueba para los dos modelos fue el test ji cuadrado de Wald. La asociación en el modelo se estableció mediante el odds ajustado $(\exp \beta)$ y su correspondiente valor de p. El nivel de confianza para establecer el modelo más apropiado fue al 95\%.

Por último, para establecer la capacidad pronóstica de los síntomas cefalea, fosfenos y epigastralgia, para predecir la clasificación de las pacientes como complicadas, se usaron el índice de Youden, las razones de verosimilitud positiva y negativa (likelihood ratios).

Los responsables del registro institucional del parto y de casos de hipertensión fueron estudiantes de medicina y enfermeras del programa de promoción y prevención institucional, quienes fueron entrenados antes de la recolección de la información. En una de las instituciones se desarrolló una prueba piloto durante 30 días para ajustar y corregir el instrumento de recolección. Uno de los investigadores realizaba control de calidad del diligenciamiento de los formatos, asegurando su integridad. 
Se hace una descripción de los tratamientos recibidos.

La información fue digitada en una base de datos construida en Microsoft Excel 8.0® y el análisis estadístico uni y bivariado en Epi Info 6.04d® y multivariado en SPSS $8.0 @$. El análisis de validez diagnóstica se realizó con Epi Dat 3.0®.

\section{RESULTADOS}

Se detectaron 89 casos de hipertensión del embarazo de 1.697 partos institucionales, para una incidencia de 5,2\% (IC 95\% 4,2-6,3\%). En todos los casos fue posible recolectar información.

\section{Características de las hipertensas}

La edad promedio de las hipertensas fue de 25 años (DE 6,5); el 7,9\% fue menor de 18 años y 9\% mayor de 38. El 8,8\% tuvo menos de dos controles prenatales, el 11,3\% entre dos y tres; el 15\% entre cuatro y cinco y el $65 \%$ más de cinco.

El 46\% eran primíparas y el 4,5\% multíparas con más de cuatro gestaciones. El 3,5\% tuvo parto antes de las 32 semanas, 5,8\% entre las 32 y 34 semanas, $16,3 \%$ entre las 35 y 36 semanas y $74,4 \%$ mayor que 37 semanas de gestación.

El 69\% de las gestantes hipertensas tuvo parto por cesárea. El 26,8\% de las hipertensas tuvo un hijo de menos de $2.500 \mathrm{~g}$; el $51,2 \%$ entre $2.500 \mathrm{y}$ $3.499 \mathrm{~g} ; \mathrm{y}$ el 22,1\% de más de $3.500 \mathrm{~g}$.

Como antecedentes personales: ocho $(9,0 \%)$ refirieron antecedente de preeclampsia, ocho con antecedente de obesidad o diabetes mellitus. Se encontró diabetes u obesidad como antecedente familiar en 19 gestantes (21,3\%).

\section{Comparación entre hipertensas y no hipertensas}

Esta comparación se consigna en la tabla 1.

\section{Criterios de clasificación de las hipertensas}

Como pacientes no complicadas se clasificaron $34,8 \%$ hipertensas sin proteinuria, 4,5\% preeclampsia moderada, 2,2 \% hipertensión crónica, para un subtotal de 47,7\%.
El 52,3\% de las gestantes hipertensas se clasificó como complicadas; el 14,6\% sin proteinuria con complicaciones; el 40,4\% con preeclampsia grave y el 3,4\% con hipertensión crónica y preeclampsia sobreagregada.

El 57,3\% presentó cefalea, 27\% fosfenos y 42,7\% epigastralgia. El 43,8\% de las hipertensas tuvieron al menos un registro de presión arterial mayor de 160/ 110, el 10,1\% trombocitopenia (recuento de plaquetas menor de 100.000/mm), 9\% enzimas hepáticas mayores de $70 \mathrm{mU} / \mathrm{L}, 22,5 \%$ deshidrogenasa láctica mayor de $500 \mathrm{mU} / \mathrm{L}, 4,5 \%$ creatinina mayor de 1,2 $\mathrm{mg} / \mathrm{dl}$ y 7,9\% presentó convulsiones.

En el 82\% no se detectó complicación clínica. El 7,9\% tuvo anemia o hemorragia, 6,7\% síndrome HELLP (2,2\% complicado) y un caso $(1,1 \%)$ de edema pulmonar, muerte materna y sospecha de HELLP.

Entre las 35 pacientes que no reportaron síntomas hubo trece complicadas, dos de ellas convulsionaron.

El compromiso perinatal se estableció en 14,6\% de los partos: síndrome de dificultad respiratoria en ocho casos $(8,9 \%)$, oligoamnios en tres $(3,5 \%)$, restricción de crecimiento en uno $(1,1 \%)$ y muerte perinatal en uno $(1,1 \%)$.

Los días de hospitalización materna oscilaron entre 1 y 15 , en cinco (6\%) pacientes fue superior a siete días. La hospitalización de los recién nacidos varió entre 1 y 30 días y en cuatro (4,8\%) fue mayor de siete días.

Se registraron datos de tensión arterial al egreso de 73 pacientes, de las cuales, 17 (23,3\%) reportaron persistencia de la hipertensión.

\section{Tratamiento}

En la tabla 2 se resume el uso estratificado de sulfato de magnesio y de tratamientos antihipertensivos por hipertensión complicada o no. El 42\% no recibió tratamiento anti-hipertensivo inicial ni de mantenimiento.

En las pacientes con complicación no se empleó sulfato de magnesio en bolos iniciales en $65,4 \%$ ni de mantenimiento en 34,6\%. Estas 
hipertensas complicadas no recibieron dosis iniciales de antihipertensivos en un 27,45\% de los casos ni de mantenimiento en el 24\%. El 15,3\% de las hipertensas con complicaciones no recibió ninguno de estos tratamientos.

\section{Pronóstico de complicación u hospitalización}

Al estudiar las variables sociodemográficas, de antecedentes personales o familiares, o antecedentes gineco-obstétricos, ninguna mostró aso-

Tabla 1. Características de embarazadas hipertensas y

de la población general de gestantes atendidas por parto en Villavicencio. Agosto - Noviembre 2003

\begin{tabular}{lccc} 
Característica & Hipertensas & No hipertensas & Valor de p \\
\hline Edad $<18$ & $7(7,86 \%)$ & $178(16,32 \%)$ & 0,038 \\
\hline Edad $>37$ & $8(9 \%)$ & $73(6,7 \%)$ & 0,43 \\
\hline G1 & $41(46,1 \%)$ & $478(46,6 \%)$ & 0,99 \\
\hline Parto $<35$ sem. & $14(15,7 \%)$ & $58(4,2 \%)$ & 0,000001 \\
\hline Cesáreas & $58(70 \%)$ & $589(34,75 \%)$ & 0,000000 \\
\hline Peso <2.500 g & $20(22,5 \%)$ & $89(7,4 \%)$ & 0,0000005 \\
\hline
\end{tabular}

ciación con la aparición de complicación o días de hospitalización.

Se encontró asociación entre hospitalización materna mayor de cuatro días y edad gestacional menor de 35 semanas, proteinuria grave (mayor de $100 \mathrm{mg} / \mathrm{dl}$ o $2 \mathrm{~g} / \mathrm{d})$ y epigastralgia. Fueron predictores de hipertensión complicada: gestación de menos de 34 semanas al momento de la hospitalización y proteinuria (mayor de $30 \mathrm{mg} / \mathrm{dl}$ o $300 \mathrm{mg} / \mathrm{d}$ ) (tabla 3).

La epigastralgia se constituyó en el síntoma de mejor pronóstico de la hipertensión complicada con un índice de Youden de 0,31, una razón de probabilidades positiva de 2,29 y negativa de 0,58 . La presencia de al menos uno de los tres síntomas solo mejora la verosimilitud negativa con 0,53 (tabla 4).

\section{DISCUSIÓN}

La incidencia de trastornos hipertensivos encontrada coincide con lo reportado en la literatura, con varia-

Tabla 2. Uso de sulfato de magnesio y tratamiento antihipertensivo en gestantes hipertensas

\begin{tabular}{l|c|c|c|c|c|c|c}
\multicolumn{7}{c}{ Uso de sulfato de magnesio } \\
\hline \multirow{2}{*}{ Estado } & \multirow{2}{*}{$\mathbf{n}$} & \multicolumn{3}{|c|}{ Dosis inicial } & \multicolumn{3}{c}{ Dosis mantenimiento } \\
\cline { 3 - 9 } & & NO & $<\mathbf{4 g}$ & $>\mathbf{4 g}$ & No & $\mathbf{1 g} / \mathbf{h}$ & $\mathbf{2 g} / \mathbf{h}$ \\
\hline No complicadas & 37 & 31 & 5 & 1 & 27 & 8 & 2 \\
\hline Complicadas & 52 & 34 & 13 & 5 & 18 & 25 & 9 \\
\hline Total & 89 & $65(73 \%)$ & $18(20,2 \%)$ & $6(6,7 \%)$ & $45(50,6 \%)$ & $33(37,1 \%)$ & $11(12,4 \%)$ \\
\hline
\end{tabular}

\begin{tabular}{|c|c|c|c|c|c|c|c|}
\hline \multicolumn{8}{|c|}{ Dosis inicial de antihipertensivo } \\
\hline \multirow{2}{*}{ Estado } & \multirow{2}{*}{$\mathbf{n}$} & \multirow{2}{*}{ NO } & \multicolumn{3}{|c|}{ Nifedipino } & \multirow{2}{*}{ AlfaMD } & \multirow{2}{*}{ Otros } \\
\hline & & & $20 \mathrm{mg}$ & $10 \mathrm{mg}$ & Sublingual & & \\
\hline No complicadas & 37 & 22 & 3 & 8 & 2 & 2 & 0 \\
\hline Complicadas & 51 & 15 & 3 & 22 & 2 & 2 & 7 \\
\hline Total & 88 & $37(42 \%)$ & $6(6,8 \%)$ & $30(34,1 \%)$ & $4(4,5 \%)$ & $4(4,5 \%)$ & $5(8 \%)$ \\
\hline \multicolumn{8}{|c|}{ Dosis mantenimiento de antihipertensivo } \\
\hline \multirow{2}{*}{ Estado } & \multirow{2}{*}{$\mathbf{n}$} & \multirow{2}{*}{ NO } & \multicolumn{2}{|c|}{ Nifedipino } & \multirow{2}{*}{ AlfaMD } & \multirow{2}{*}{ Otros } & \\
\hline & & & >30/día & $\leq 30 /$ día & & & \\
\hline No complicadas & 37 & 23 & 3 & 9 & 2 & 0 & \\
\hline Complicadas & 51 & 14 & 9 & 19 & 2 & 7 & \\
\hline Total & 88 & $37(42,0 \%)$ & $12(13,6 \%)$ & $28(31,8)$ & $4(4,5 \%)$ & $7(8,0 \%)$ & \\
\hline
\end{tabular}


Tabla 3. Factores asociados con hipertensión complicada u hospitalización mayor de 4 días en gestantes con hipertensión

\begin{tabular}{|c|c|c|c|}
\hline Covariables & AOR & IC 95\% & p \\
\hline \multicolumn{4}{|c|}{ Asociación con hipertensión complicada } \\
\hline Gestación $<34$ semanas & 7,96 & $1,85-34,29$ & 0,0053 \\
\hline $\begin{array}{l}\text { Proteinuria }>30 \mathrm{mg} / \mathrm{dl} \mathrm{o} \\
300 \mathrm{mg} / 24 \mathrm{~h}\end{array}$ & 20,11 & $3,76-107,53$ & 0,0005 \\
\hline \multicolumn{4}{|c|}{ Asociación con hospitalización $>4$ días } \\
\hline Edad gestacional $<35$ semanas & 3,51 & $1,51-8,15$ & 0,0035 \\
\hline $\begin{array}{l}\text { Proteinuria grave }>100 \mathrm{mg} / \mathrm{dl} \\
\text { o } 2 \mathrm{~g} / 24 \mathrm{~h}\end{array}$ & 7,02 & $1,53-32,22$ & 0,0121 \\
\hline Epigastralgia & 4,26 & $1,02-17,78$ & 0,0471 \\
\hline
\end{tabular}

tensas y no hipertensas, lo que controvierte su consideración como factores de riesgo. ${ }^{3,13}$ La proporción de neonatos con peso menor de $2.000 \mathrm{~g}$ fue significativamente mayor en el grupo de hipertensas que en la población general, sin una relación de este efecto con un grupo de edad específico.

Entre los antecedentes estudiados y que han sido descritos como importantes en la génesis de la preeclampsia, ninguno pudo relacionarse de manera válida con las complicaciones. Es de resaltar el efecto protector del antecedente familiar de obesidad o diabetes, que podría indicar mejores condiciones nutricionales y por ende, mayor protección de complicaciones.

Los hallazgos clínicos y paraclínicos significaron resultados acordes con los descritos, la proteinuria desde el primer rango que se considera positiva es factor de riesgo bien definido para la aparición de complicaciones y parece sustentar la probabilidad de que el recién nacido tenga mayores periodos de hospitalización. ${ }^{10,11}$

Los síntomas demostraron capacidad variable para pronosticar complicaciones o mayores periodos de hospitalización, excepto la epigastralgia que presentó asociación significativa con todas las variables. La hemólisis se ha reconocido como un indicador de compromiso hepático ${ }^{14,15}$ y en el estudio demuestra serlo al correlacionarse de manera notoria con los niveles de DHL mayores de $500 \mathrm{mU} / \mathrm{L}$. Ningún otro criterio diagnóstico para el síndrome de HELLP (plaquetas o transaminasas) mostró esta asociación.

Las pacientes hipertensas con síndrome de HELLP fueron 6,74\% (IC 95\% 1,5-6,9\%) que representó el 15,38\% de las mujeres con hipertensión complicada, lo que supera lo establecido de 4 a 12\% entre pacientes con preeclampsia severa $^{14}$ y a lo registrado en el Instituto Materno Infantil de Bogotá, donde se reportó en 15\% de preeclámpticas. ${ }^{16}$ En la Clínica San Pedro Claver 
de Bogotá ${ }^{15}$ se tuvo una proporción mayor de HELLP entre los casos de trastornos hipertensivos del embarazo, con un registro de 10,6\%, posiblemente por su condición de centro de referencia.

En cuanto a los tratamientos empleados, se encontró el empleo de dosis inferiores a las recomendadas, incluso en pacientes clasificadas como complicadas. ${ }^{3,4,13}$ Debe reconocerse la limitación de los recursos terapéuticos para la hipertensión de la embarazada impuesta por el Sistema General de Seguridad en el país, pero de ninguna manera justifican el altísimo porcentaje de partos intervenidos a los que son sometidas estas pacientes, convirtiendo la cesárea en el principal y en muchas veces el único tratamiento empleado.

Aunque se carece de cifras sobre muertes perinatales relacionadas con la hipertensión en el embarazo, una muerte de un recién nacido se debe considerar menor a la esperada, por lo que se puede asumir que se está realizando una intervención positiva relacionada con la calidad de atención neonatal. El impacto sobre la mortalidad materna requiere de estudios más prolongados, la estadística acumulada para el 2003 registra tres casos, ${ }^{17}$ lo que también sugeriría el impacto positivo de las unidades de cuidado especializado.

El registro institucional de casos de hipertensión arterial en el embarazo facilitó la monitorización real de un evento de interés en salud pública, dadas sus condiciones de magnitud, gravedad, posibilidad de prevención e interés poblacional. La sistematización de una acción de vigilancia en salud pública permitió obtener información permanente de la situación del fenómeno estudiado.

\section{AGRADECIMIENTOS}

Al Hospital Departamental, Clínica LlanosSaludCoop, Clínica Los Centauros-Servimédicos, Clínica Meta, Clínica Martha y Clínica Carlos Hugo Estrada Castro de la ciudad de Villavicencio por su colaboración.

\section{REFERENCIAS}

1. Cunningham FG, Gant N, et al. Hypertensive disorder in pregnancy. En: Williams Obstetrics. $21^{\text {st }}$ edition. New York: Mc Graw Hill; 2001. p. 567-618.

2. Conde-Agudelo A, Belizan JM. Risk factors for preeclampsia in a large cohort of Latin-American and Caribbean women. BJOG 2000;107:75-83.

3. Cifuentes R. Preeclampsia-eclampsia: diagnóstico y manejo. En: Cifuentes R. Ginecología y obstetricia basadas en las evidencias. Bogotá DC: Editorial Distribuna; 2002. P. 439-44.

4. Bautista A. Hipertensión y embarazo. Toxemia gravídica. En: Ñañez H, Ruiz AI, eds. Texto de Obstetricia y Perinatología. Una contribución a la enseñanza del arte, ciencia y tecnología. Bogotá DC: Pharmacia Upjohn; 1999. p 487-530.

5. Report of the National High Blood Pressure Education Program: Working Group Report on High Blood Pressure in Pregnancy. Am J Obstet Gynecol 2000;183:S1-S22.

6. Esplin MS, Fausett MB, Fraser A, Kerber R, Mineau G, Carrillo J, et al. Paternal and maternal components of the predisposition to preeclampsia. N Engl J Med 2001;344:867-72.

7. Odegard RA, Vatten LJ, Nilsen ST, Salvesen KA, Austgulen R. Risk factors and clinical manifestations of preeclampsia. BJOG 2000; 107:1410-6.

8. Sibai BM, Ewell M, Levine RJ, Klebanoff MA, Esterlitz J, Catalano PM, et al. Risk factors associated with preeclampsia in healthy nulliparous women. The Calcium for Preeclampsia Prevention (CPEP) Study Group. Am J Obstet Gynecol 1997;177:1003-10.

9. Pridjian G, Puschett JB. Preeclampsia. Part 1: clinical and pathophysiologic considerations. Obstet Gynecol Surv 2002;57:598-618.

10. Chesley LC. Diagnosis of preeclampsia. Obstet Gynecol 1985;65:423-5.

11. Ferrazzani S, Caruso A, De Carolis S, Martino IV, Mancuso S. Proteinuria and outcome of 444 pregnancies complicated by hypertension. Am J Obstet Gynecol 1990;162:366-71.

12. Sibai BM. The HELLP syndrome (hemolysis, elevated liver enzymes, and low platelets) much ado about nothing? Am J Obstet Gynecol 1990;162:311-6.

13. Ministerio de Salud. Guías de atención de las complicaciones hipertensivas asociadas al embarazo. Bogotá, DC; 2000. 
14. Sibai BM, Taslimi MM, el-Nazer A, Amon E, Mabie BC, Ryan GM. Maternal-perinatal outcome associated with the syndrome of hemolysis, elevated liver enzymes, and low platelets in severe preeclampsia-eclampsia. Am J Obstet Gynecol 1986;155:501-9.

15. Martínez F, Quintero S, Zapata S. Síndrome HELLP: descripción de 100 casos en Bogotá-Colombia 1999. Rev Colomb Obstet Ginecol 2001;52:323-30.
16. Bautista A, Ruiz A. Síndrome HELLP un enemigo silente. En: Nañez H, Ruiz AI, et al. Obstetricia y perinatología. Bogotá DC: Pharmacia \& Upjohn; 1999. p. 487-530.

17. Secretaría Departamental de Salud del Meta. Enfermedades Sujetas a Vigilancia Intensificada. Villavicencio; 2003. 